\title{
The Reduction of Catheter-Related Blood Stream Infections through the Implementation of an Interdisciplinary Healthcare Team
}

\author{
Kristen Scatliffe, ${ }^{1}$ Adebanke Davis, ${ }^{2}$ Carla Wang-Kocik, ${ }^{1}$ Nelson Medina Villanueva, ${ }^{3}$ \\ Maria Espiritu-fuller, ${ }^{1}$ Renita Larang, ${ }^{1}$ Patricia Dimitriou, ${ }^{1}$ Amy Doran, ${ }^{1}$ Anne Repayo, ${ }^{1}$ \\ Jeremias Murillo, ${ }^{1}$ Christian A. Engell, ${ }^{1}$ Morris Cohen, ${ }^{1}$ and Jennifer A. LaRosa ${ }^{1}$ \\ ${ }^{1}$ Newark Beth Israel Medical Center, USA \\ ${ }^{2}$ Dartmouth Hitchcock Medical Center, USA \\ ${ }^{3}$ Florida Hospital Memorial Medical Center, Bert Fish Medical Center and Halifax Medical Center, USA
}

Correspondence should be addressed to Jennifer A. LaRosa; jenniferlarosa@yahoo.com

Received 22 August 2014; Revised 17 December 2014; Accepted 13 January 2015

Academic Editor: Ricardo Rivera-Fernández

Copyright (C) 2015 Kristen Scatliffe et al. This is an open access article distributed under the Creative Commons Attribution License, which permits unrestricted use, distribution, and reproduction in any medium, provided the original work is properly cited.

\begin{abstract}
In December 2012, a multidisciplinary task force was implemented to address the elevated number of central line associated boodstream infections (CLABSIs) at Newark Beth Israel Medical Center from January 2012 to December 2012. Sixty-eight CLABSIs were documented within the adult inpatient population, resulting in a rate of 14.7 CLABSIs/1,000 central line days in the adult inpatient population. This was well above the national average of 1.87 infections per 1,000 central line days. Most of these infections were noted to be within the critical care units where the rate was at 2.86 CLABSIs/1,000 central line days. However, in 2013, the annual rate was decreased to 0.709 CLABSIs $/ 1000$ line days $(P<0.001)$ with similar trends observed across the critical care units. Analysis of CLASBI data indicates that the implementation of a multidisciplinary task force dedicated to appropriate central line insertion, maintenance, and the removal of unnecessary central venous catheters can have an impact on reducing rates of CLASBIs throughout the adult inpatient population, including those within critical care units.
\end{abstract}

\section{Background}

Hospital associated infections (HAIs) are defined by the CDC as "infections that patients develop while they are receiving care in a health care setting for another condition with the estimated incidence of HAIs in the United States in 2012 being 4.5 per 100 patients" [1]. In 1950, the American Hospital Association recommended that hospitals begin surveillance for HAIs due to a surge in infections caused by Staphylococcus aureus seen in postoperative patients. Today, bloodstream infections represent 14\% [1] of all HAIs in the United States. Most of these bloodstream infections have been associated with central venous catheters (CVCs), which are defined by the National Healthcare Safety Network (NHSN) as "intravascular catheters that terminate at or close to the heart or in one of the great vessels which is used for infusion, withdrawal of blood, or hemodynamic monitoring" [2]. In the US alone, the associated cost attributed to HAIs in 2012 was approximately 28-33 billion dollars [3]. HAIs continue to be a significant source of morbidity and mortality in the US Healthcare system. Bloodstream infections are divided into two categories: central line associated bloodstream infections (CLABSIs) and catheter-related bloodstream infections (CRBIs). The term CLABSI refers primarily to bloodstream infections in a patient with a central line in place within the 48-hour period before onset of the bloodstream infection that is not related to infection at another site. CRBIs, on the other hand, are bloodstream infections that require specific laboratory testing to identify the catheter as the origin of the infection; this testing is not a part of the criteria for a CLABSI. It is estimated that 41,000 CLABSIs occur in U.S. hospitals annually with an associated cost of $\$ 16,550.47$ per infection and an increased length of stay [4]. These infections have been associated with an estimated inpatient mortality rate of 
$12-25 \%$ [5]. For the purposes of surveillance, the definition of a CLABSI is more practical, but it comes with its own limitations. It has been noted by the Joint Commission that this definition may overestimate the actual rate of infections from central lines as opposed to another remote site.

In 2012, at Newark Beth Israel Medical Center (NBIMC), a total number of 68 CLABSIs were recorded in the adult population, and the resulting total represented a figure above the national benchmark. This elevated number of infections prompted the question, "what can we do as a teaching institution to reduce the rate of these bloodstream infections?" In 2011, in the Clinical Journal of Infectious Disease, Mermel et al. made an important note that the pathogenesis of catheter infections is a multifactorial process where both intraluminal and extraluminal causes must be investigated [6]. For this reason, we focused on multiple strategies targeted at reduction. A multidisciplinary CLABSI prevention task force was formed to examine our practices with regard to central line insertion, maintenance, and removal, and to implement a stringent policy that promoted tight adherence to CDC practice bundle elements with a unified approach that involved physicians and nurses actively monitoring each hospital unit. The objective of this paper is to evaluate the impact the implementation of a multidisciplinary task force dedicated to appropriate central line insertion can have on reducing rates of CLASBIs.

\section{Methods}

This study took place at NBIMC, a single center site with an urban patient population, from January 2012 to December 2013. This teaching hospital is university-affiliated and has 673 beds and approximately 23,000 annual admissions. This facility employs 149 full-time physicians and 96 part-time physicians, along with 855 full-time registered nurses and 239 part-time nurses.

All patients admitted to any adult unit, including the Intensive Care Units, from January 2012 to December 2013 were included in this study. The control period was January through December 2012, and the intervention period, after development of the CLABSI Task Force Prevention Team, was from January through December 2013. Patients who were seen solely on an outpatient basis were not included in this study (e.g., outpatient hemodialysis and individuals receiving chemotherapy). Patients admitted to the inpatient population of NBIMC but who did not receive a central line during their admission, or who were admitted before January 2012 and after December 2013, were also not included. CLABSIs were defined as recovery of a pathogen from a blood culture (a single blood culture for organisms not commonly present on the skin) in a patient who had a central line at the time of infection or within the 48-hour period before development of infection. The infection could not be related to any other infection the patient might have and must not have been present or incubating when the patient was admitted to NBIMC. All CLASBIs were documented.

Immediately prior to the intervention period, a multidisciplinary task force was formed which consisted of administrators, representatives from Infection Control and
Infectious Disease Departments, nurse and physician representatives, and "champions" from every hospital unit, which included critical care. The composition of the task force was based on the recommended guidelines by the CDC, which state that "each member of the care team should have a stake in the outcome, and all the members should have clear roles and responsibilities, a shared vision, and a common purpose to achieve the valued goals" [7]. The roles of each member of the multidisciplinary task force were as follows.

(i) ICU Director led interdisciplinary rounds and queried each of the practitioners as to their specialty area.

(ii) Infectious Control representatives and Infectious Disease attendings gave information about whether active infections were considered to be community versus hospital acquired and whether or not infections were related to catheters.

(iii) The nursing staff provided the front line presentation of the patient's active medical problems, current evidence of infection, and daily white blood cell count and fever curve. From this group, hospital unit champions were elected.

(iv) Pulmonary Critical Care attendings and fellows ensured that all physicians performing central lines were adequately trained and became accredited in CVC insertion. Accreditation was achieved by performing 10 assisted central line insertions with supervision from any accredited physician.

Initially, the interdisciplinary task force met weekly starting in December 2012, and each unit was assigned champions [8] who were responsible for reviewing every central line inserted, promoting the removal of unnecessary lines, and conducting a review of any potential CLABSIs (positive blood cultures in patients with central lines).

The use of evidence-based insertion and maintenance bundles was paramount to the success of the task force initiative and helped to standardize central line care within all units of NBIMC. Bundles were defined previously as "groupings of best practices with respect to a disease process that individually improve care, but when applied together result in substantially greater improvement" [9]. The science supporting the bundle of components is sufficiently established to be considered the standard of care [9]. The CVC insertion bundle used at NBIMC included the following best practices: hand hygiene, the use of Aseptic technique (which includes maximal barrier protection and preparation of the skin with chlorhexidine, specifically $2 \%$ concentration), the use of ultrasound guidance with all line insertions, the placement of a biopatch [10-13] at all line entry points, avoidance of the femoral vein for use as an insertion site, and prompt removal of all CVCs not deemed unnecessary by the task force. The maintenance bundle used at NBIMC was based on a previous construct from the CDC 2002 guidelines which includes: assessing the continued need for the catheter every day, performing catheter site care with chlorhexidine at dressing changes and changing gauze dressings every 2 days and clear dressings every 7 days (and more frequently 
if soiled, damp, or loose). Using a $2 \%$ chlorhexidine for daily skin cleansing was established by Munoz-Price et al. after they evaluated the effect of chlorhexidine bathing of patients on the rate of CLABSI in a 70-bed long-term acute care hospital [14]. Their work demonstrated that this intervention reduces the rate of CLASBI from 9.5 to 3.8 per 1,000 catheter days. Also included in our maintenance bundle was the replacement of administration sets and add-on devices no more frequently than every 72 hours unless contamination occurred. Replacing tubing used to administer blood, blood products, or lipids within 24 hours of start of infusion was also monitored, along with changing IV port protectors no more often than 72 hours (or according to manufacturer's recommendations and whenever the administration set was changed).

A part of the task force initiative included thorough education of all team members on best practices, which was accomplished through several mediums including the following: weekly meetings with all members of the task force initiative; informational displays visible on every adult inpatient unit, including the critical care floors; and mandated certification in central line insertion for all physicians, including residents and fellows. Hand washing was emphasized to all staff members of NBIMC through the CINCO (clean in and clean out) protocol. CINCO is a pinnacle part of the insertion bundle, as it should be conducted prior to palpating the site of catheter insertion, before and after inserting the catheter, and before and after accessing, replacing, repairing, or dressing the catheter.

2.1. Data Collection. A retrospective analysis of data from January 2012 to December 2012 was conducted. Data from January to December 2013 was collected and analyzed. The data collected during both periods included the date of admission, date of the first positive culture, organism confirmed on culture, the site of the central line, and evidence of compliance with bundles. During data collection, each CLABSI was specified by the organism present on culture and grouped by their Gram stain and morphology, as previously done by other examples in the literature [15]. Compliance with bundle elements was monitored through three forms: central line insertion note, nursing checklist, and time out form. A copy of the nursing checklist and insertion note is included in this discussion. The central line note was used as a tool to verify that physicians inserting these lines documented the location of the inserted line and that the elements of the insertion bundle were completed effectively. The nursing checklist was designed with an additional focus-it required the nurse to verify that insertion and maintenance best practices were occurring with every line. If the procedure was compromised in anyway, as in whether a time out was not performed or maximal sterile barrier precautions were compromised, the nurse also would ensure that the insertion would be stopped. Overall, adult rates of CLABSIs hospital-wide, CLABSIs in all critical care areas, and CLABSIs that occurred exclusively in medical/surgical Intensive Care unit were collected. All three areas were compiled to show the rate per quarter of CLABSIs within all adult units at NBIMC.
TABLE 1: Number of CLABSIs in the adult population at NBIMC.

Interventional period Jan. 2012-Dec. 2013 Jan. 2013-Dec. 2013

Total number of

CLABSIs in all adult

68

18

units

Total number of

CLABSIs in the critical

care units

32

Total number of

CLABSIs in the

medical/surgical units

TABLE 2: Number of CLABSIs within critical care units.

\begin{tabular}{lcc}
\hline Units & $\begin{array}{c}\text { Number of } \\
\text { CLABSIs in 2012 }\end{array}$ & $\begin{array}{c}\text { Number of } \\
\text { CLABSIs in 2013 }\end{array}$ \\
\hline Medical surgical ICU & 15 & 0 \\
Cardiothoracic ICU & 5 & 2 \\
Coronary care unit & 12 & 5 \\
\hline
\end{tabular}

TABLE 3: Location of central venous catheters in CLABSI patients.

\begin{tabular}{lcc}
\hline Sites of lines & $\begin{array}{c}\text { Quantity seen in } \\
\text { 2012 CLABSI } \\
\text { patients }\end{array}$ & $\begin{array}{c}\text { Quantity seen in } \\
\text { 2013 CLABSI } \\
\text { patients }\end{array}$ \\
\hline Internal jugular & 31 & 9 \\
Subclavian & 24 & 9 \\
Femoral & 5 & 0 \\
External jugular & 0 & 1 \\
Unknown & 19 & 0 \\
\hline
\end{tabular}

2.2. Statistical Analysis. Data were expressed as means and standard deviations (SD) for continuous variables and percentages for qualitative variables. We used Student's $t$-test for comparisons of two means and the Chi-square test to compare proportions. Critical alpha was set to $P<0.05$.

\section{Results}

The number of CLABSI cases within the adult population by unit at NBIMC for the pre- and postintervention periods is provided in Table 1 . The highest number of CLABSIs in the preintervention period was observed in the Medical Surgical ICU $(n=36)$, which represents $22 \%$ of all central lineassociated bloodstream infections seen in 2012 at NBIMC (see Table 2). This percentage is not surprising, as the ICU is the focus of many major landmark studies centered on reduction strategies [16]. This focus on ICU units is due to the fact that while bloodstream infections can occur in multiple demographics; CLABSIs with organisms that have antimicrobial resistance occur more within the ICU than in the general inpatient population [17]. The sites of line placement are provided in Table 3. A statistically significant decrease in the overall number of CLABSIs from the preintervention period $(M=14.7 / 1000$ line days $)$ to the postintervention period 


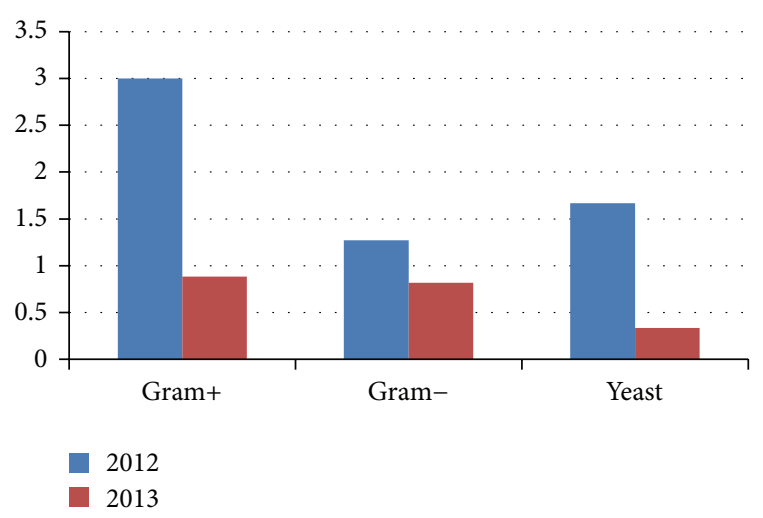

FIGURE 1: Mean number of CLASBI organisms present before and after intervention.

$(M=0.709 / 1000$ line days $)$ was demonstrated $t(22)=4.118$, $P<0.001$.

Due to the polymicrobial makeup of the positive cultures seen within this study and the multifactorial nature of CLABSI pathogenesis, we simplified the data as most previous landmark articles did and grouped the organisms based on their general Gram stain characteristics and morphology. At NBIMC during the pre- and postintervention periods, CLABSIs were mostly caused by Gram-positive microorganisms $(72.85 \%$ and $60 \%$, resp.; see Figure 1). The primary organisms reported by the CDC as causative pathogens in 2012 included coagulase-negative Staphylococci, Staphylococcus aureus, enterococci, and Candida spp. [15] Per data from the SCOPE database (Surveillance and Control of Pathogens of Epidemiological Importance), Gram-negative bacilli accounted for approximately $20 \%$ of CLABSIs reported to the CDC in 2011 [15]. In comparing the trends seen within our study to those reported by the CDC, it is noted that $21 \%$ of the samples were positive for Staphylococcus epidermidis, $10 \%$ were Methicillin sensitive Staph aureus, $15.7 \%$ were Enterococcus, and 7\% were of the genus Candida. Other cultures seen in a smaller percentage included P. aeruginosa, Klebsiella pneumoniae, and E. coli.

Results of analysis indicate that there was a statistically significant decrease in the mean number of Gram-positive CLABSIs from the preintervention to postintervention periods $t(32)=-2.038, P=0.050$. The mean number of yeast CLABSIs also decreased significantly in the postintervention period, $t(4)=-2.828, P=0.047$. Although there was a decrease in the mean number of Gram-negative CLABSIs from pre- to postintervention periods, the decrease was not significant $(P=0.377)$. These findings can be explained by the fact that the Gram-positive and Gram-negative sources of infection are directly addressed with the implementation of bundle strategies that emphasize the use of maximal sterile barrier precautions and chlorhexidine $2 \%$ preparation for the skin prior to insertion. CLABSIs secondary to fungal organisms are typically secondary to immunosuppression, prolonged presence of the central venous catheters, and are typically seen in surgical and ICU patients, all of which are risk factors not decreased by these bundle elements. Of note, the number of line days between 2012 and 2013 actually increased within the non-ICU population from 13,902 days to 14,601 days, which could have given rise to an increase in yeast CLABSIs. The ICU population, in contrast, saw a reduction of line days from 11,178 days to 10,768 days.

Compliance to time out, $\chi^{2}(2, N=84)=6.459, P=$ 0.040 , and central venous line insertion notes, $\chi^{2}(3, N=$ $84)=9.601, P=0.022$, improved significantly between 2012 and 2013. There was no significant difference in compliance with the completion of the nursing checklist measure from the pre- to postintervention periods $P=0.115$. When looking at the overall mean compliance scores, there was a significant difference between 2012 and 2013, $t(81)=2.191$, $P=0.0313$. Overall compliance improved significantly in 2013 as compared to 2012. The overall compliance scores also predicted the rates of infection for 2013, $R^{2}=.344, F(1,10)=$ 5.249, $P=0.045$, but not for 2012, $P=0.418$.

\section{Discussion}

The results of this study demonstrate that the implementation of a multidisciplinary CLABSI prevention task force reduced the rates of infection from an average of 2.7359 infections per month to 0.7071 infections per month in 2013. This success can be attributed to the implementation of the multidisciplinary task force that focused on implementing evidence-based strategies based on 2011 CDC guidelines and ensuring compliance with these measures. While maximal sterile barrier precautions and the use of chlorhexidine $0.5 \%$ have been shown to reduce infections in prior studies, these strategies alone do not explain the dramatic improvement in the rates of infection between pre- and postintervention periods in our study. Without the task force addressing the issue of education, it is predicted that these results would be less dramatic and even more so less permanent $[18,19]$. When providers are not appropriately educated in best practices and sterile techniques, this increases the risk for insertionrelated contamination from improper skin decontamination and breaks in the sterile field, an increased number of skin punctures, and increased manipulation of the catheter during insertion, all of which increase the risk of catheter-related bloodstream infections [20]. Therefore, one of the requirements of providers at NBIMC is that they become certified in central venous catheter insertion. This certification is granted specifically to providers that display competence in insertion of CVCs and have performed insertion of at least 10 central venous catheters using ultrasound guidance [13] under direct supervision from a certified attending or fellow. All central venous catheters inserted during this study period were performed by physicians who were accredited in central venous catheter insertion.

Another strategy that had a profound impact on the rates of infection was the completion of a time out prior to procedure and a central line insertion note was completed after insertion of a central venous catheter. Nurses are central in hospital efforts to improve quality care and "empowering the nurse who is present at bedside, to lead the bundle implementation, increased personal ownership and compliance and ultimately improved practice and patient outcomes in previous examples from the literature" $[7,9]$. 


\title{
-n BARNABAS HEALTH a Newark Beth Israel Medical Center
}

\author{
Central Venous Line Insertion Note
}

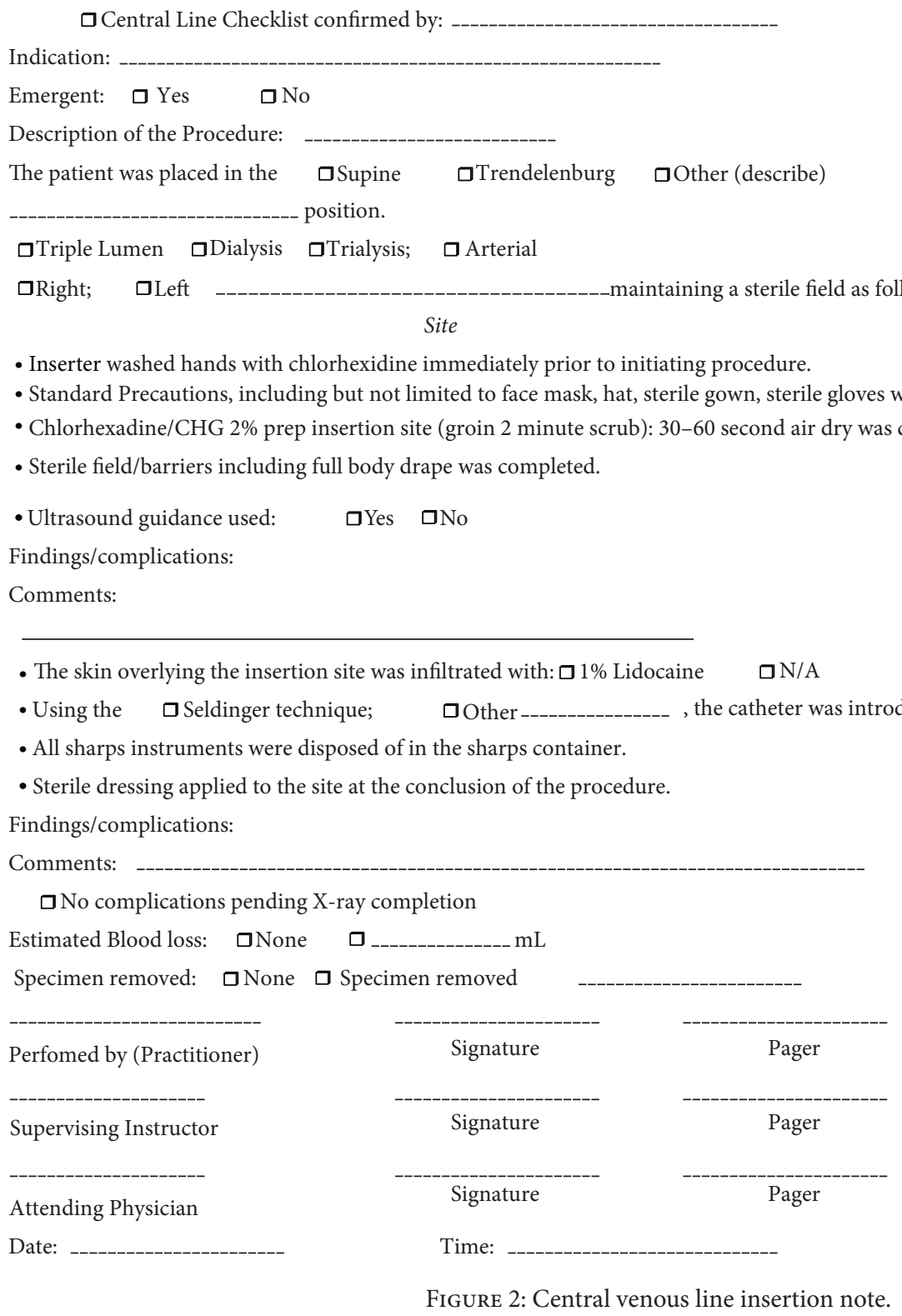

Burton et al. remarked in JAMA 2009 that MRSA infections traditionally accounted for $50 \%$ of all CRBIs seen within the critical care unit, but they reiterated that the landscape of causative pathogens continues to change based mostly on antimicrobial resistance, among other factors [21]. Therefore, we expected that most of the CLABSIs observed would be positive for MRSA; however, our data demonstrates that the primary organism during the preintervention period at NBIMC in the critical care unit was Staphylococcus epidermis.
This was the anticipated trend for the preintervention period. This was not surprising, as the presence of Staphylococcus epidermis suggested that the normal skin flora was introduced during the insertion of the line; the organisms observed indicate the mechanism of pathogenesis. The prevalence of Staphylococcus epidermis is easily explained by the mechanism of organism migration via insertion through the skin into the cutaneous catheter tract. It is the most common route of infection for short-term catheters per a 1991 American 


\section{- BARNABAS HEALTH Newark Beth Israel Medical Center}

\section{Children's Hospital of New Jersey}

Nursing Checklist for Central Venous Catheter Insertion:

Purpose: To evaluate current infection prevention practices and identify opportunities for improvement.

When: During all central venous or central arterial line insertions or re-wires

By whom: Primary Nurse or Charge Nurse

If there is an observed violation of infection control practices, line placement should stop immediately and the violation should be corrected. If a correction is required, mark Yes to \#13

(1) Sulfa allergies? $\square$ No $\square$ If Yes (use non-impregnated catheter)

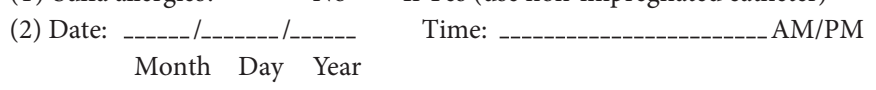

(3) Indication: $\square$ Multiple Drugs/Drips $\quad \square$ TPN $\square$ No peripheral access $\square$ Other

(4) Location: $\square$ Room \# -------- $\square$ Cath Lab $\square$ ER $\square$ Interventional Radiology $\square$ OR

(5) Procedure: $\square$ New Central Line Placement $\quad \square$ Rewire $\quad \square$ Arterial Line $\square$ Other

(6) Site: $\square$ Subclavian $\square$ Jugular $\square$ Umbilical

(7) Type of CVC: $\quad \square$ TLC $\quad \square$ Swan Ganz $\quad \square$ Cordis $\quad \square$ Dialysis $\quad \square$ Portacath $\square$ PICC

(8) Is the procedure: $\square$ Elective

(9) Who was the primary inserter? $\square$ Intensivist $\square$ Attending $\square$ Fellow $\square$ Resident $\square$ Intern

$\square$ Anesthesia $\square$ Other

(10) Central line insertion checklist utlized noted in the Universal

Protocol Documentation

(11) Before the procedure, did the inserter:

Perform a Time out

Wash Hands with clorhexidine

Was hand washing directly observed?

For age 2 months and older:

Prep insertion site with $2 \%$ CGH-30 second,

scrub (groin-2 min scrub) and 30-60 second air dry.

For age less than 2 months or patients that are allergic to chlorhexadine:

Prep insertion site with $70 \%$ alcohol swab from center to periphery

Using a circular motion for a total of three times, allow to dry and then

Prep insertion site with povodine iodine form center to periphery

using a circular motion three times around site followed by

greater than $30 \mathrm{~s}$. dry time.

Sterile field/field barriers including full body drape

and additional draping as needed.

(12) During the procedure, did the inserter:

Use sterile Gloves

Use hat, mask, sterile gown?

Maintain a sterile field?

Did all personnel assisted (within 3 feet)

follow the above precautions:

Ultrasound guidance-was it available?

If Ultrasound guidance was available-was it used?

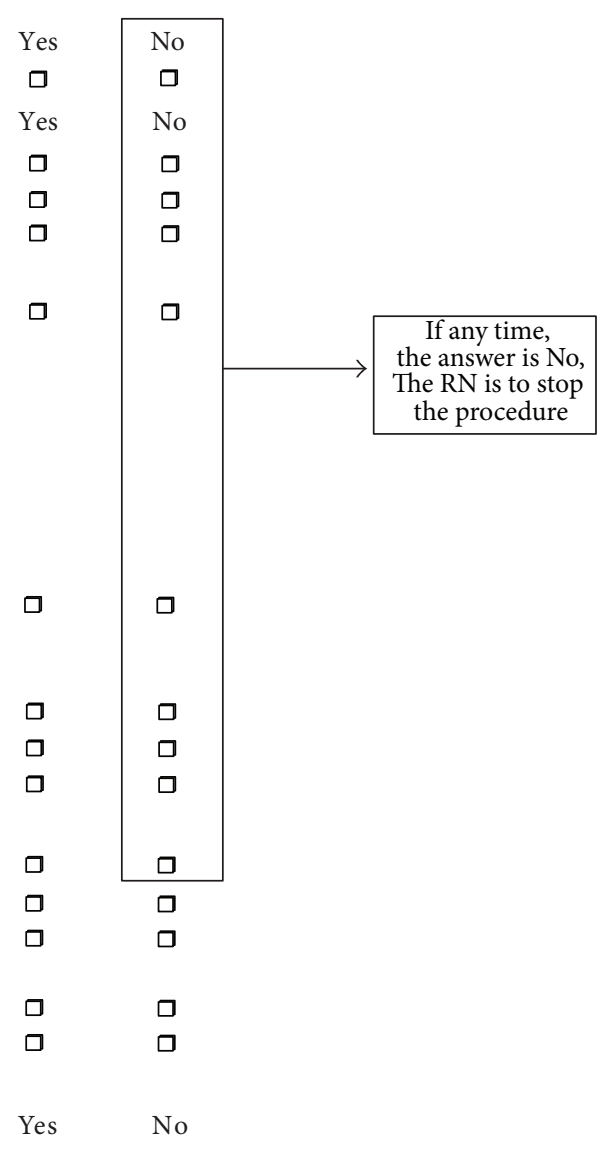

(13) After the procedure:

Was a sterile dressing applied to the site?

Was a procedure note written?

(14) Was a correction required to ensure compliance with infection control procedures (please circle)

Comments:

Signature RN: 
Journal of Medicine study on pulmonary artery catheters by Mermel et al. [22].

The present study has certain limitations, which include lack of blinding to intervention allocation and the multidisciplinary task force itself, which may be considered by some smaller single centers as a complex intervention. The establishment of a task force may cause critical care units to believe that they will be reliant on dedication from at least one expert advocate with sufficient experience in management of CLABSI reduction, or at least infection control, to have the same results. Although this study was conducted at a single center, potentially limiting external validity, it should be noted that our interventions can be broadly implemented. Where most other single center studies fail to show theoretical or empirical evidence to support widespread intervention, our findings are strongly supported by several existing landmark articles addressing strategies designed to reduce the rate of central line associated bloodstream infections. The interventions implemented within our study are merely representative of a combination of the existing best practices into one functional construct supported by a multidisciplinary team, which decreases the risk of failure in comparison to prior strategies where one protagonist was responsible for motivating and ensuring adherence to bundle elements. As previously mentioned, compliance to the nursing checklist did not reach statistical significance. Nonadherence to the nursing checklist and compliance with this portion of the protocol were later addressed by the multidisciplinary task force and nursing management. Another limitation was the lack of good documentation as it pertains to the sites of central venous catheter insertion. Initially, we wanted to examine the difference between rates of infections and insertion locations of the central venous catheters. However, because of the lack of reporting in this area, we were unable to assess the significance of this element at our center.

In 2012 during the preintervention study period, the greatest number of CLABSIs hospital-wide occurred during the month of September 2012, which could be explained by the lack of training within new providers (new intern classes are unfamiliar with bundle elements and correct technique) inserting central venous catheters, decreased nursing involvement, and a lack of strict adherence to proven best practices. Future studies would benefit from the inclusion of multiple hospital sites to increase the power of the data. In addition, identifying how a multidisciplinary task force may affect other components of patient care (e.g., ventilator days and proper nutrition within the ICU) would be worthy areas of exploration.

\section{Appendix}

See Figures 2 and 3.

\section{Conflict of Interests}

The authors declared no potential conflict of interests with respect to the research, authorship, and/or publication of this paper.

\section{References}

[1] US Department of Health and Human Services, "HHS Action Plan to Prevent Healthcare-Associated Infections: Introduction," 2012, http://www.hhs.gov/ash/initiatives/hai/introduction.html.

[2] US Centers for Disease Control and Prevention, "National Healthcare Safety Network," 2012, http://www.cdc.gov/nhsn/ PDFs/pscManual/4PSC_CLABScurrent.pdf.

[3] R. Scott, "The direct medical costs of healthcare-associated infections in US hospitals and the benefits of prevention," Tech. Rep., US Centers for Disease Control and Prevention, 2009, http://www.cdc.gov/hai/pdfs/hai/scott_costpaper.pdf.

[4] United States Centers for Disease Control and Prevention, "Vital signs: central line-associated blood stream infectionsUnited States, 2001, 2008, and 2009," Morbidity and Mortality Weekly Report, vol. 60, no. 8, pp. 243-248, 2011.

[5] R. M. Klevens, J. R. Edwards, C. L. Richards Jr. et al., "Estimating health care-associated infections and deaths in U.S. Hospitals, 2002," Public Health Reports, vol. 122, no. 2, pp. 160-166, 2007.

[6] L. A. Mermel, M. Allon, E. Bouza et al., "Clinical practice guidelines for the diagnosis and management of intravascular catheter-related infection. 2009 Update by the infectious diseases society of America," Clinical Infectious Diseases, vol. 49, no. 1, pp. 1-45, 2009.

[7] R. G. Hughes, Ed., Patient Safety and Quality: An EvidenceBased Handbook for Nurses, Agency for Healthcare Research and Quality, U.S. Department of Health and Human Services, Rockville, Md, USA, 2008, http://archive.ahrq.gov/professionals/clinicians-providers/resources/nursing/resources/nurseshdbk/nurseshdbk.pdf.

[8] P. W. Stone, E. C. Hedblom, D. M. Murphy, S. B. Miller, and L. Homan, "The economic impact of infection control: making the business case for increased infection control resources," American Journal of Infection Control, vol. 33, no. 9, pp. 542-547, 2005.

[9] Institute for Healthcare Improvement, "Implement the IHI Central Line Bundle," 2012, http://www.ihi.org/knowledge/ Pages/Changes/ImplementtheCentralLineBundle.aspx.

[10] L. A. Mermel and B. Hudson, "Prevention of intravascular catheter-related infections," Annals of Internal Medicine, vol. 132, no. 5, pp. 391-402, 2000.

[11] N. P. OGrady, M. Alexander, L. A. Burns et al., "Guidelines for the prevention of intravascular catheter-related infections," Clinical Infectious Diseases, vol. 52, no. 9, pp. e162-e193, 2011.

[12] J. Marschall, L. A. Mermel, D. Classen et al., "Strategies to prevent central line-associated bloodstream infections in acute care hospitals," Infection Control and Hospital Epidemiology, vol. 30, no. 8, p. 815, 2009.

[13] M. Pittiruti, H. Hamilton, R. Biffi, J. MacFie, and M. Pertkiewicz, "ESPEN Guidelines on Parenteral Nutrition: central venous catheters (access, care, diagnosis and therapy of complications)," Clinical Nutrition, vol. 28, no. 4, pp. 365-377, 2009.

[14] L. S. Munoz-Price, B. Hota, A. Stemer, and R. A. Weinstein, "Prevention of bloodstream infections by use of daily chlorhexidine baths for patients at a long-term acute care hospital," Infection Control and Hospital Epidemiology, vol. 30, no. 11, pp. 1031-1035, 2009.

[15] H. Wisplinghoff, T. Bischoff, S. M. Tallent, H. Seifert, R. P. Wenzel, and M. B. Edmond, "Nosocomial bloodstream infections in US hospitals: analysis of 24,179 cases from a prospective 
nationwide surveillance study," Clinical Infectious Diseases, vol. 39, no. 3, pp. 309-317, 2004.

[16] B. Allegranzi, S. B. Nejad, C. Combescure et al., "Burden of endemic health-care-associated infection in developing countries: systematic review and meta-analysis," The Lancet, vol. 377, no. 9761, pp. 228-241, 2011.

[17] P. Pronovost, D. Needham, S. Berenholtz et al., "An intervention to decrease catheter-related bloodstream infections in the ICU," New England Journal of Medicine, vol. 355, no. 26, pp. 27252732, 2006.

[18] D. M. Lin, K. Weeks, C. G. Holzmueller, P. J. Pronovost, and J. C. Pham, "Maintaining and sustaining the on the CUSP: stop BSI model in Hawaii," Joint Commission Journal on Quality and Patient Safety, vol. 39, no. 2, pp. 51-60, 2013.

[19] J. M. Walz, R. T. Ellison III, D. A. Mack et al., “The bundle 'plus': the effect of a multidisciplinary team approach to eradicate central line-associated bloodstream infections," Anesthesia \& Analgesia, 2013.

[20] N. P. O’Grady, R. N. Mary Alexander, L. A. Burns et al., "Guidelines for the Prevention of Intravascular Catheter-Related Infections, 2011," CCOC Research Group. The Bundle 'Plus': The Effect of a Multidisciplinary Team Approach to Eradicate Central Line-Associated Bloodstream Infections. April 2011.

[21] D. C. Burton, J. R. Edwards, T. C. Horan, J. A. Jernigan, and S. K. Fridkin, "Methicillin-resistant Staphylococcus aureus central line-associated bloodstream infections in US intensive care units, 1997-2007," The Journal of the American Medical Association, vol. 301, no. 7, pp. 727-736, 2009.

[22] L. A. Mermel, R. D. McCormick, S. R. Springman, and D. G. Maki, "The pathogenesis and epidemiology of catheterrelated infection with pulmonary artery swan-ganz catheters: a prospective study utilizing molecular subtyping," American Journal of Medicine, vol. 91, no. 3, pp. 197S-205S, 1991. 


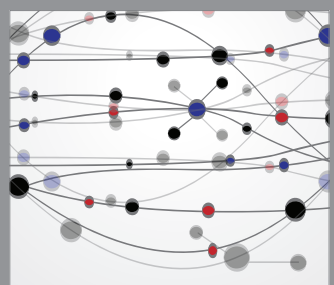

The Scientific World Journal
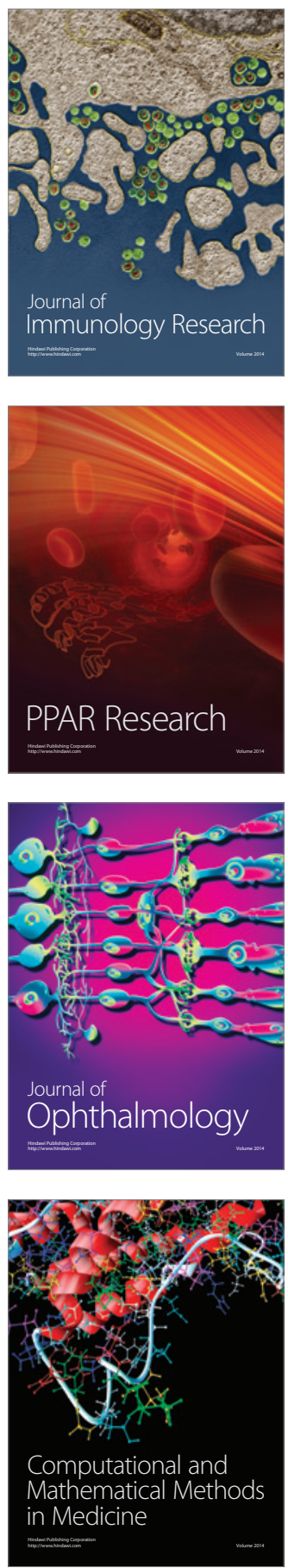

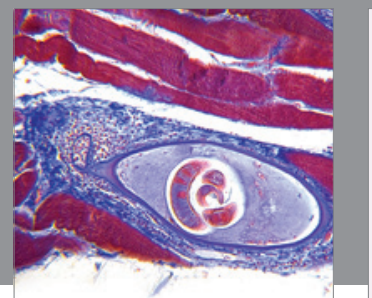

Gastroenterology

Research and Practice
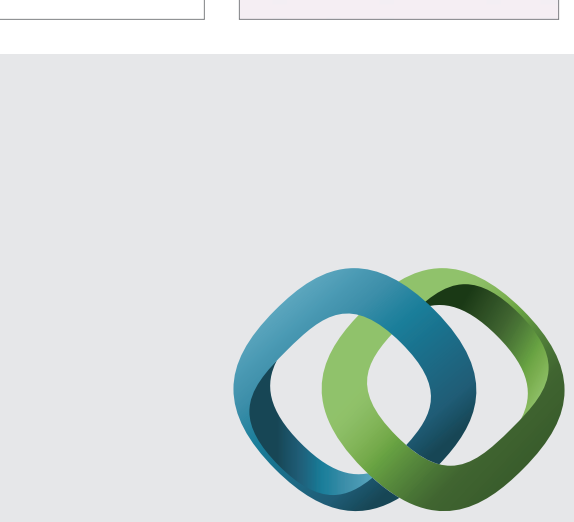

\section{Hindawi}

Submit your manuscripts at

http://www.hindawi.com
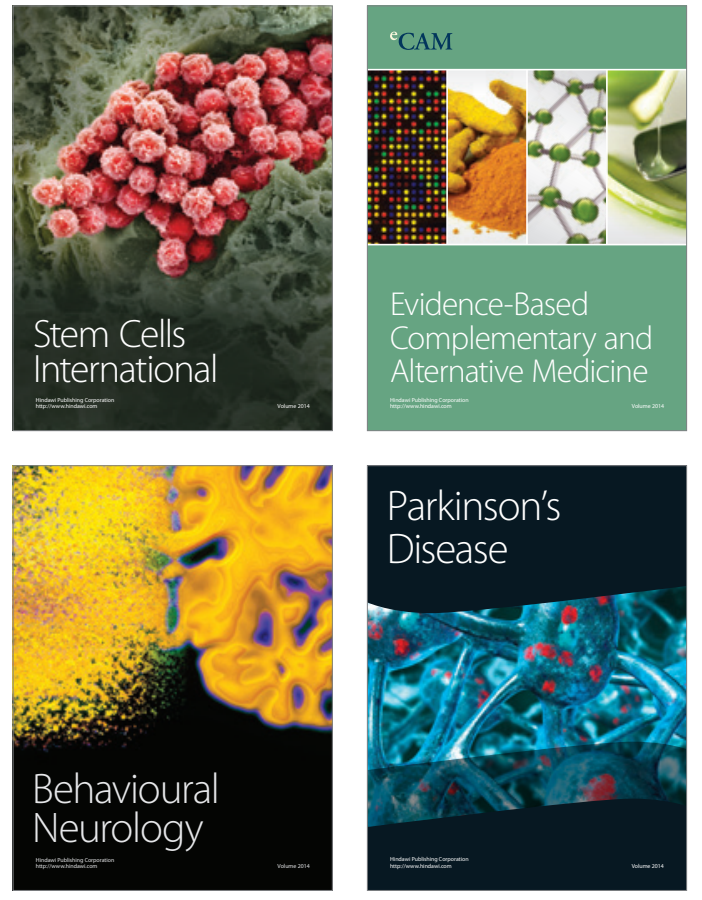
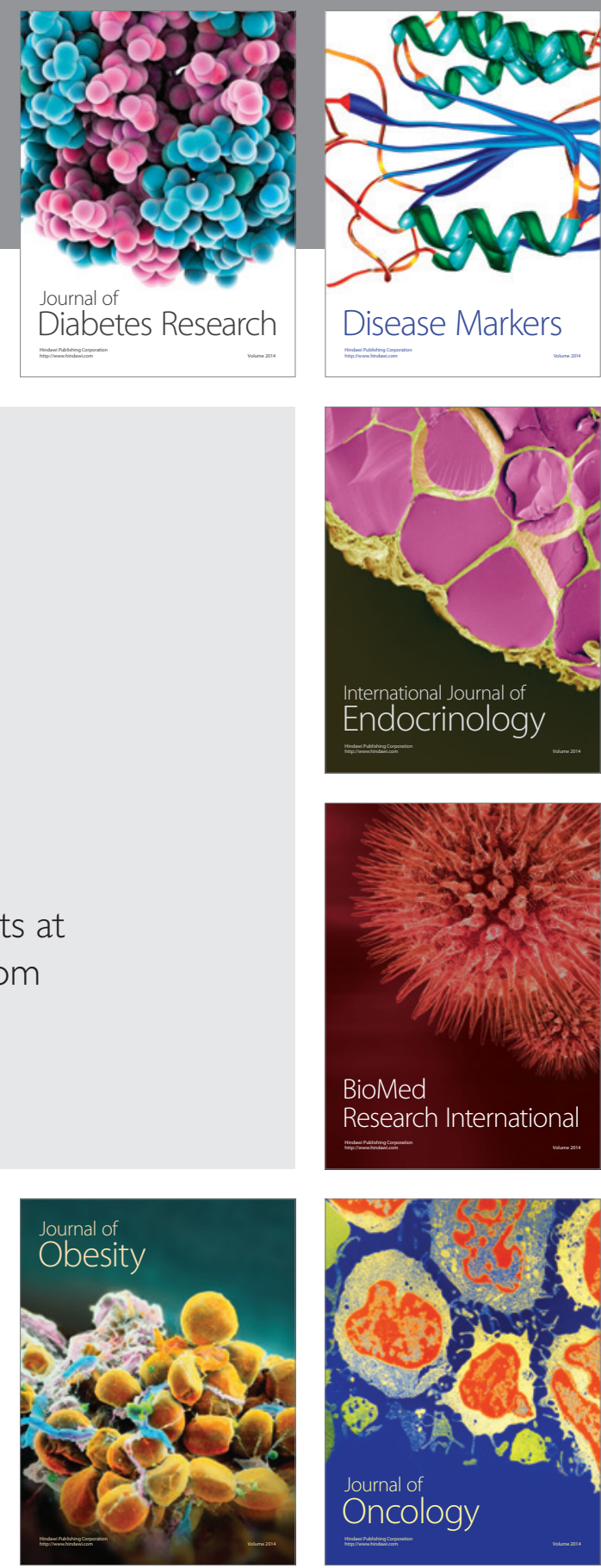

Disease Markers
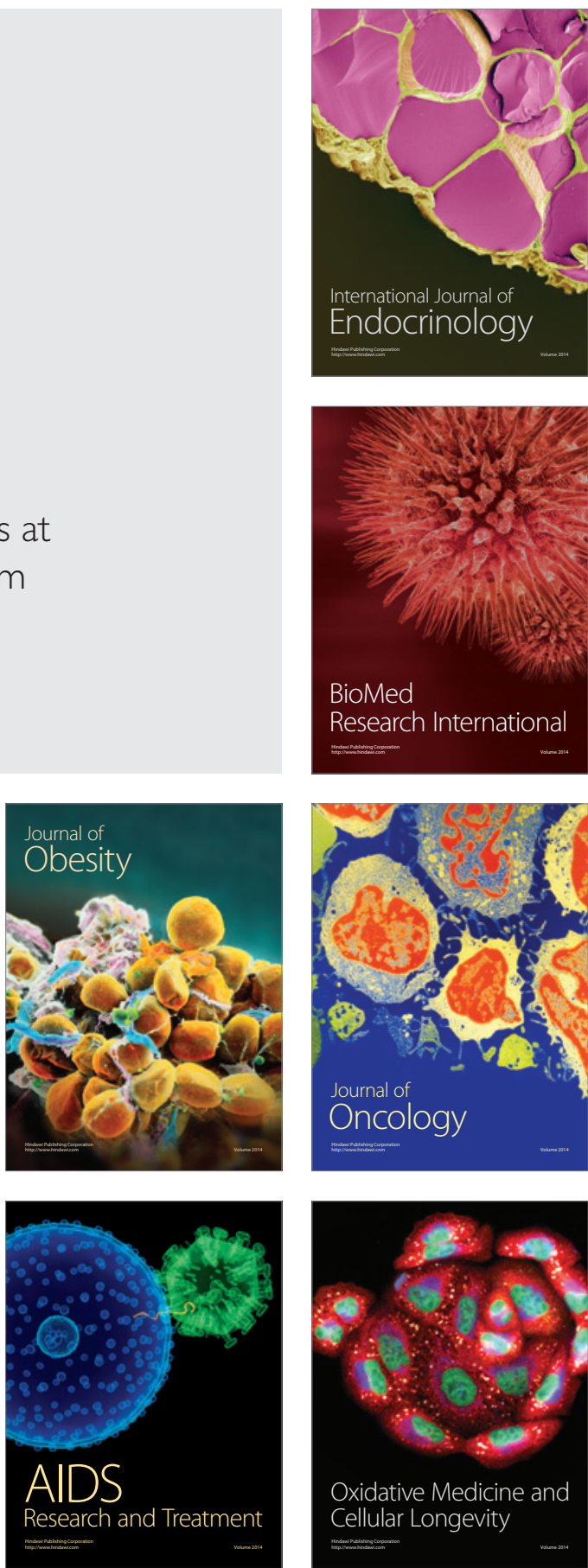